\title{
THE FACTORIZATION OF $M$-CHANNEL FIR and IIR COSINE-MODULATED FILTER BANKS AND THEIR MULTIPLIER-LESS REALIZATIONS USING SOPOT COEFFICIENTS
}

\author{
S. C. Chan, J. S. Mao, P. M. Yiu, and K. L. Ho \\ Department of Electrical and Electronic Engineering \\ The University of Hong Kong, Pokfulam Road, Hong Kong.
}

\begin{abstract}
This paper proposes a new factorization for the $M$-channel perfect reconstruction (PR) IIR Cosine-Modulated filter banks (CMFB) proposed previously by the authors. This factorization, which is based on the lifting scheme, is also complete for the PR FIR CMFB as well as the general two-channel PR IIR filter banks if the determinant of the polyphase matrix is equal to constant multiplies of signal delays. It can be used to convert a numerically optimized nearly PR CMFB to a structurally PR system. Furthermore, the arithmetic complexity of the FB using this structure can be reduced asymptotically by a factor of two. When the forward and inverse transforms are implemented with the same set of SOPOT coefficients, a multiplier-less CMFB can be obtained. Its arithmetic complexity is further reduced and it becomes very attractive for VLSI implementation.
\end{abstract}

\section{INTRODUCTION}

The cosine modulated filter banks (CMFB) [1] or the Extended Lapped Transforms [2] are efficient structures for implementing M-channel PR filter banks. They have high stopband attenuation, and low design and implementation complexities. The design of biorthogonal CMFB is usually performed by constrained non-linear optimization $[7,8]$. Unlike the orthogonal case where it is possible to perform an unconstrained optimization on the coefficients of the lossless lattice structure [1], the filter banks so obtained are in general pseudo PR. To overcome this problem, factorizations of the filter bank, which structurally enforces the PR condition, is necessary. In [9], the polyphase components of the CMFB are parameterized by the lifting scheme so that the filter bank is still PR for different choices of the lifting coefficients. The design problem can then be formulated as an unconstrained optimization problem with the lifting coefficients as variables. Factorizations of arbitrary-delay and arbitrary-length FIR CMFB were also obtained recently by Schuller [5] and Gao et al. [6]. In this paper, a new factorization for the IIR CMFB, which was previously obtained by the authors in [3], is proposed. This factorization, which is based on the lifting scheme [4], is also complete for the PR FIR CMFB as well as the general two-channel PR IIR filter banks if the determinant of the polyphase matrix is equal to constant multiplies of signal delays. Since the unconstrained optimization using the lifting coefficients results in highly nonlinear objective function, we prefer to formulate the design problem as a constrained optimization in the filter coefficients. The proposed factorization technique is then applied to convert it to a structurally PR system. Like the lifting scheme, it also reduces the arithmetic complexity of the filter bank asymptotically by a factor of two. Further, by representating the coefficients in the lifting structure as SOPOT coefficients and using the SOPOT DCT-IV in [10], multiplier-less biorthogonal CMFBs with low complexity can be obtained. The paper is arranged as follows: Section II is devoted to the proposed factorization for two-channel PR IIR FBs. Its application to $M$ channel IIR CMFB is described in Section III followed by several examples in Section IV. Conclusions are drawn in Section V.

II. FACTORIZATION OF TWO CHANNEL PR IIR FBS

Let the IIR analysis filters $H_{0}(z)$ and $H_{1}(z)$ be written as $H_{i}(z)=N_{i}(z) / D_{i}(z)$ for $i=0,1$, where $N_{i}(z)$ and $D_{i}(z)$ are respectively the numerator and denominator polynomials of $H_{i}(z)$. Multiplying the numerator and denominator of $H_{i}(z)$ by $D_{i}(-z)$, we have

$$
H_{i}(z)=\frac{N_{i}(z) D_{i}(-z)}{D_{i}(z) D_{i}(-z)}=\frac{\tilde{N}_{i}(z)}{\widetilde{D}_{i}\left(z^{2}\right)}, i=0,1 .
$$

Decomposing the numerators $\tilde{N}_{0}(z)$ and $\tilde{N}_{1}(z)$ into their polyphase components: $\quad \tilde{N}_{0}(z)=\tilde{N}_{00}\left(z^{2}\right)+z^{-1} \tilde{N}_{01}\left(z^{2}\right)$, and $\tilde{N}_{1}(z)=\widetilde{N}_{10}\left(z^{2}\right)+z^{-1} \widetilde{N}_{11}\left(z^{2}\right)$, the polyphase components of $H_{i}(z)$ are determined as follows

$$
\begin{aligned}
H_{i}(z) & =\frac{\widetilde{N}_{i 0}\left(z^{2}\right)}{\widetilde{D}_{i}\left(z^{2}\right)}+z^{-1} \frac{\widetilde{N}_{i 1}\left(z^{2}\right)}{\widetilde{D}_{i}\left(z^{2}\right)}, i=0,1 . \\
& =H_{i 0}\left(z^{2}\right)+z^{-1} H_{i 1}\left(z^{2}\right)
\end{aligned}
$$

For perfect reconstruction (PR), the determinant of the polyphase matrix $\boldsymbol{E}(z)$ can be chosen as constant multiplies of signal delay. This yields

$$
\widetilde{N}_{00}(z) \widetilde{N}_{11}(z)-\widetilde{N}_{10}(z) \tilde{N}_{01}(z)=\beta \cdot z^{-d} \widetilde{D}_{0}(z) \tilde{D}_{1}(z) .
$$

Equation (2-3) can be viewed as an ideal $\left\{\phi_{0}: \phi_{0}(z)=A(z) \tilde{N}_{00}(z)-B(z) \tilde{N}_{01}(z)=\beta \cdot z^{-d} \tilde{D}_{0}(z) \tilde{D}_{1}(z)\right\}$

generated by $\tilde{N}_{00}(z)$ and $-\tilde{N}_{01}(z)$. The Highest Common Factor (HCF) of $\tilde{N}_{00}(z)$ and $\tilde{N}_{01}(z)$ must therefore divide $\phi_{0}(z)=z^{-d} \widetilde{D}_{0}(z) \widetilde{D}_{1}(z)$. For simplicity, it is assumed that such factors have already been extracted from $\tilde{N}_{00}(z)$ and $\tilde{N}_{01}(z)$, and they are co-prime to each other. The general solutions of $\widetilde{N}_{10}(z)$ and $\widetilde{N}_{11}(z)$ are

$$
\tilde{N}_{10}(z)=\hat{N}_{10}(z)-Q(z) \tilde{N}_{00}(z),
$$

and $\quad \tilde{N}_{11}(z)=\hat{N}_{11}(z)-Q(z) \tilde{N}_{01}(z)$,

for some $\hat{N}_{10}(z)$ and $\hat{N}_{11}(z)$ in the ideal $\phi_{0}(z)$, and some polynomial $Q(z)$ in $F[z]$. The general solution of $\tilde{N}_{1}(z)$ is:

$$
\tilde{N}_{1}(z)=\hat{N}_{1}(z)-Q\left(z^{2}\right) \tilde{N}_{0}(z),
$$

where $\hat{N}_{1}(z)=\hat{N}_{10}\left(z^{2}\right)+z^{-1} \hat{N}_{10}\left(z^{2}\right)$. Dividing both sides of $(2-5)$ by $\widetilde{D}_{1}\left(z^{2}\right)$, one gets

$$
H_{1}(z)=\hat{H}_{1}(z)-\hat{Q}\left(z^{2}\right) H_{0}(z),
$$

where $\hat{Q}(z)=Q(z) \cdot \tilde{D}_{0}(z) / \widetilde{D}_{1}(z)$ is an arbitrary causal stable rational function and $\hat{H}_{1}(z)=\hat{N}_{1}(z) / \tilde{D}_{1}\left(z^{2}\right)$ is a particular solution. (2-6) allows us to parameterize all solutions of the PR IIR filter banks using $\hat{Q}(z)$ and $\hat{N}_{1}(z)$. The latter can be constructed from the Euclidean algorithm or lifting steps as in [4]. Suppose that $\widetilde{N}_{00}(z)$ and $\widetilde{N}_{01}(z)$ are coprime and apply Euclidean algorithm to them yields

$$
\left[\begin{array}{c}
\tilde{N}_{00}(z) \\
\tilde{N}_{01}(z)
\end{array}\right]=\prod_{i=1}^{n}\left[\begin{array}{cc}
q_{i}(z) & z^{-d_{1}} \\
1 & 0
\end{array}\right] \cdot\left[\begin{array}{c}
K z^{-d_{0}} \\
0
\end{array}\right],
$$


where $q_{i}(z)$ are polynomials in $F[z], K$ is a non-zero constant, and $d_{i}$ are positive integers. The delay $z^{-d_{i}}$ results from the removal of the lowest order coefficients, instead of the higher order coefficients in conventional polynomial division, during the polynomial division, i.e. they are treated as Laurent polynomials. If the highest order coefficients are removed as in conventional polynomial division, $d_{i}=0$. This offers more flexibility in carrying out the Euclidean algorithm than using ordinary polynomial, which helps to reduce the dynamic range of $q_{i}(z)$. It should be noted that this factorization is highly non-unique. Dividing both sides of (2-7) by $\widetilde{D}_{0}(z)$ gives

$$
\left[\begin{array}{l}
H_{00}(z) \\
H_{01}(z)
\end{array}\right]=\prod_{i=1}^{n}\left[\begin{array}{cc}
q_{i}(z) & z^{-d_{i}} \\
1 & 0
\end{array}\right] \cdot\left[\begin{array}{c}
K z^{-d_{0}} / \widetilde{D}_{0}(z) \\
0
\end{array}\right] \text {. }
$$

Using (2-8), the following polyphase matrix $\boldsymbol{P}(z)$ with the required determinant $\beta \cdot z^{-d}$ can be formed.

$$
\begin{aligned}
\boldsymbol{P}(z) & =\left[\begin{array}{cc}
H_{00}(z) & \hat{H}_{10}(z) \\
H_{01}(z) & \hat{H}_{11}(z)
\end{array}\right] \\
& =\prod_{i=1}^{n}\left[\begin{array}{cc}
q_{i}(z) & z^{-d_{i}} \\
1 & 0
\end{array}\right] \cdot\left[\begin{array}{cc}
K z^{-d_{0}} / \widetilde{D}_{0}(z) & 0 \\
0 & (-1)^{n} \beta \cdot z^{-\left(d-d_{i}\right)} \widetilde{D}_{0}(z) / K
\end{array}\right],
\end{aligned}
$$

where $d_{c}$ is the sum of the delays $d_{i}$ and it should be smaller than $d$. Otherwise, the system delay will be increased beyond the given value $2 d+1$. Using (2-6), the general solution of the IIR analysis polyphase matrix $\boldsymbol{E}(z)$ is

$$
\begin{aligned}
& \boldsymbol{E}^{T}(z)=\left[\begin{array}{cc}
H_{00}(z) & \hat{H}_{10}(z) \\
H_{01}(z) & \hat{H}_{11}(z)
\end{array}\right]\left[\begin{array}{cc}
1 & -\hat{Q}(z) \\
0 & 1
\end{array}\right] \\
& =\prod_{i=1}^{n}\left[\begin{array}{cc}
q_{i}(z) & z^{-d_{i}} \\
1 & 0
\end{array}\right] \times \\
& {\left[\begin{array}{cc}
K z^{-d_{0}} / \widetilde{D}_{0}(z) & 0 \\
0 & (-1)^{n} \beta \cdot z^{-\left(d-d_{0}\right)} \widetilde{D}_{0}(z) / K
\end{array}\right]\left[\begin{array}{cc}
1 & -\hat{Q}(z) \\
0 & 1
\end{array}\right] \text {. }}
\end{aligned}
$$

(2-10) can also be implemented as a series of lifting steps [4].

\section{FACTORIZATION OF IIR CMFB}

In CMFBs, the analysis filters $f_{k}(n)$ and the synthesis filters $g_{k}(n)$ are derived from a prototype filter $h(n)$ by cosine modulation,

$$
\begin{aligned}
& f_{k}(n)=h(n) \cos \left(\omega_{k} n+\varphi_{k}\right)=h(n) c_{k . n}, \\
& \text { and } \quad g_{k}(n)=h(n) \cos \left(\omega_{k} n+\hat{\varphi}_{k}\right)=h(n) \bar{c}_{k, n} \text {, } \\
& k=0,1, \ldots, M-1 \text {, }
\end{aligned}
$$

where

$$
\begin{aligned}
& c_{k, n}=\cos \left[\frac{\pi}{2 M}(2 k+1)\left(n-\frac{N-1}{2}\right)+(-1)^{k} \frac{\pi}{4}\right], \\
& \bar{c}_{k, n}=\cos \left[\frac{\pi}{2 M}(2 k+1)\left(n-\frac{N-1}{2}\right)-(-1)^{k} \frac{\pi}{4}\right],
\end{aligned}
$$

$k=0,1, \ldots, M-1, n=0,1, \ldots, N-1$, are the modulation sequences. The PR condition, in terms of the polyphase components $H_{l}(z)$ of the prototype filter is

$$
\begin{aligned}
& H_{l}(z) H_{2 M-t-1}(z)+H_{A t+1}(z) H_{M-l-1}(z)=\beta \cdot z^{-u}, \\
& \quad l=0,1, \ldots, M-1,
\end{aligned}
$$

where $\alpha$ is an integer determining the delay of the filter banks. This simplified PR condition greatly reduces the number of variables to be optimized and the implementation complexity. For the IIR CMFB in [3], the denominators of all the polyphase components are identical, and we have $H_{1}(z)=N_{1}(z) / D(z)$. The PR condition in (3-3) is simplified to

$$
\begin{aligned}
& N_{l}(z) N_{2 M-t-1}(z)+N_{M+l}(z) N_{M-l-1}(z)=\beta \cdot z^{-a} D^{2}(z), \\
& \quad l=0,1, \ldots, M-1 .
\end{aligned}
$$

Equation (3-4) can also be viewed as an ideal $\left\{\phi_{0}: \phi_{0}(z)=A(z) N_{t}(z)+B(z) N_{M+l}(z)=\beta \cdot z^{-\alpha} D^{2}(z)\right\}$

generated by $N_{l}(z)$ and $N_{M+l}(z)$. For simplicity, we shall assume that the common factors of $N_{l}(z)$ and $N_{M+l}(z)$, which must divide the right hand side of (3-4), have already been removed and they are co-prime to each other. The general solutions of $N_{2 M-l-1}(z)$ and $N_{M-l-1}(z)$ in (3-4) are given by

$$
\begin{aligned}
& N_{M-l-1}(z)=\tilde{N}_{M-l-1}(z)-Q_{l}(z) N_{l}(z), \\
& N_{2 M-l-1}(z)=\tilde{N}_{2 M-l-1}(z)+Q_{l}(z) N_{M+l}(z),
\end{aligned}
$$

for some $\widetilde{N}_{M-t-1}(z)$ and $\widetilde{N}_{2 M-i-1}(z)$ in the ideal $\phi_{0}(z)$, and some $Q(z)$ in $F[z]$. Dividing both sides of (3-5) by $D(z)$, we have

$$
\begin{aligned}
& H_{M-l-1}(z)=\widetilde{H}_{M-l-1}(z)-Q_{l}(z) H_{i}(z), \\
& H_{2 M-l-1}(z)=\widetilde{H}_{2 M-i-1}(z)+Q_{l}(z) H_{M+l}(z),
\end{aligned}
$$

and

$$
\begin{aligned}
& {\left[\begin{array}{cc}
H_{l}(z) & H_{M-l-1}(z) \\
-H_{M+l}(z) & H_{2 M-l-1}(z)
\end{array}\right]} \\
& \quad=\left[\begin{array}{cc}
H_{l}(z) & \widetilde{H}_{M-l-1}(z) \\
-H_{M+l}(z) & \widetilde{H}_{2 M-l-1}(z)
\end{array}\right]\left[\begin{array}{cc}
1 & -Q_{l}(z) \\
0 & 1
\end{array}\right]
\end{aligned}
$$

$\widetilde{H}_{M-l-1}(z)$ and $\widetilde{H}_{2 M-l-1}(z)$ are particular solution to (3-3). Since $N_{l}(z)$ and $N_{M+l}(z)$ are coprime, they can be written, by using the Euclidean algorithm, as

$$
\left[\begin{array}{c}
N_{l}(z) \\
-N_{M+t}(z)
\end{array}\right]=\prod_{i=1}^{n_{t}}\left[\begin{array}{cc}
q_{i}^{(l)}(z) & z^{-d_{i}^{\prime \prime \prime}} \\
1 & 0
\end{array}\right] \cdot\left[\begin{array}{c}
K_{l} z^{-d_{0}} \\
0
\end{array}\right],
$$

where $K_{l}$ is a non-zero constant. Dividing both sides of (3-8) by $D(z)$, we have

$$
\left[\begin{array}{c}
H_{i}(z) \\
-H_{A t+1}(z)
\end{array}\right]=\prod_{i=1}^{n_{1}}\left[\begin{array}{cc}
q_{i}^{(\prime \prime}(z) & z^{-\left(i^{\prime \prime}\right.} \\
1 & 0
\end{array}\right] \cdot\left[\begin{array}{c}
K_{1} z^{-d_{i \prime}} / D(z) \\
0
\end{array}\right] .
$$

Then, a particular solution for $\widetilde{H}_{k t-i-1}(z)$ and $\tilde{H}_{2 M-l-1}(z)$ can be constructed as follows

$$
\begin{aligned}
& {\left[\begin{array}{cc}
H_{1}(z) & \widetilde{H}_{M-t-1}(z) \\
-H_{M+t}(z) & \widetilde{H}_{2,1-t-1}(z)
\end{array}\right]}
\end{aligned}
$$

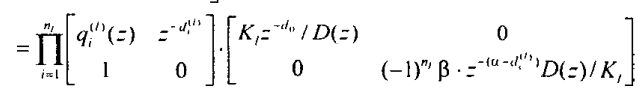

where $\beta$ and $d_{c}^{(\prime)}$ are respectively the scale factor and the sum of the delays generated during the division of the Laurent polynomials. Combining (3-7) and (3-10), the desired solution is

$$
\begin{aligned}
& {\left[\begin{array}{cc}
H_{1}(z) & H_{M-t-1,1}(=) \\
-H_{A+t+1}(\xi) & H_{2, i t-t-1}(\xi)
\end{array}\right]}
\end{aligned}
$$

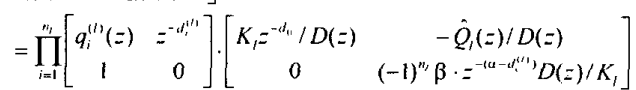

$$
\begin{aligned}
& l=0,1, \ldots, M-1,
\end{aligned}
$$

where $\hat{Q}_{l}(z)=K_{i} Q_{i}(z)$. It should be noted that in the FIR case, $D(z)=1$, and (21) provides a complete factorization of the FIR CMFB. This factorization is also applicable to FIR CMFB with other modulation because their PR conditions are still of the form (3-3) [7]. We now consider some examples. 


\section{EXAMPLES}

Example 4.1

Factorization of 4-channel FIR biorthogonal CMFB:

In this example, a 4-channel FIR biorthogonal CMFB with length $N=2 m M=2 \times 5 \times 4=40$ was factored using the proposed factorization. It was designed by the nonlinear optimization method in [8]. The length of each polyphase component is 5 and the delay parameter $\alpha$ in (3-3) is equal to 3 . The system delay is $D=2(\alpha+1) M-1=31$ samples. To avoid large dynamic range of the coefficients in the quotients, $q_{4}^{(l)}(z),(l=0,1)$, are obtained using ordinary polynomial division, while the last one is obtained by removing the lowest powers of $z^{-1}$, i.e $z^{0}$, as well as the highest power $z^{-2}$. The remainder is $K_{l} z^{-1}$ and its quotient is denoted by $L q_{4}^{(l)}(z),(l=0,1)$. An additional delay is also added to matrix of $L q_{4}^{(l)}(z)$ (top right entry), giving the delay parameter $d_{c}^{(i)}$ in (3$11)$ a value of 2 . Finally we obtain the following

$$
\begin{aligned}
& {\left[\begin{array}{cc}
H_{7}(z) & H_{3}(z) \\
-H_{4}(z) & H_{0}(z)
\end{array}\right]} \\
& \quad=\prod_{i=0}^{3}\left[\begin{array}{cc}
q_{i}^{(0)}(z) & 1 \\
1 & 0
\end{array}\right] \cdot\left[\begin{array}{cc}
L q_{4}^{(0)} & z^{-1} \\
z^{-1} & 0
\end{array}\right] \times\left[\begin{array}{cc}
K_{0} & -Q_{0}^{\prime}(z) \\
0 & -\beta / K_{0} \cdot z^{-1}
\end{array}\right], \\
& \text { where } l=0 ; \\
& {\left[\begin{array}{cc}
H_{2}(z) & H_{6}(z) \\
-H_{1}(z) & H_{5}(z)
\end{array}\right]} \\
& \quad=\prod_{i=0}^{3}\left[\begin{array}{cc}
q_{i}^{(1)}(z) & 1 \\
1 & 0
\end{array}\right] \cdot\left[\begin{array}{cc}
L q_{4}^{(1)} & z^{-1} \\
z^{-1} & 0
\end{array}\right] \times\left[\begin{array}{cc}
K_{1} & -Q_{1}^{\prime}(z) \\
0 & -\beta / K_{1} \cdot z^{-1}
\end{array}\right], \\
& \text { where } l=1 .
\end{aligned}
$$

Detailed coefficients of the factorization are given in Table 1. Figure 1(a) shows the frequency responses of the analysis filter banks after factorization. Since the input to the polyphase components in (4-1) will be down-sampled by a factor of $2 M$, the analysis filtering requires 3.25 multiplications and 2.75 additions per sample. Direct implementing the polyphase component, on the other hand, will require 10 multiplications and 9 additions per sample. Thus, the arithmetic complexity is reduced by approximately a factor of two.

\section{Example 4.2}

Multiplier-less 4-channel FIR biorthogonal CMFB using SOPOT:

In this example, a multiplier-less 4-channel FIR birothogonal CMFB with length $N=2 m M=2 \times 5 \times 4=40$, using the factorization obtained in example 4.1 , is designed. The coefficients in $q_{i}(z)$ and $Q_{i}^{\prime}(z)$ are represented as the following sum-of-powers-of-two (SOPOT) coefficients

where

$$
\begin{aligned}
& q_{\theta}=\sum_{k=1}^{1} a_{k} 2^{b_{k}}, \\
& a_{k} \in\{-1,1\} \text { and } b_{k} \in\{-r, \ldots,-1,0,1, \ldots r\} .
\end{aligned}
$$

$r$ is the range of the coefficients and $t$ is the number of terms being used in each coefficient. Therefore, all the multiplications can be replaced by limited number of shifts and additions, making it very attractive for VLSI implementation. The SOPOT coefficients, which are obtained by genetic algorithm [10], are shown in table 2 , while the frequency responses of the analysis filters are shown in figure 1(b).

\section{Example 4.3}

Factorization of Low-Delay 4-channel IIR PR CMFB:

In this example, the low-delay IIR CMFB in [3] is factored using the proposed algorithm. The orders of the numerator $N_{k}(z)$ and the denominator $D(z)$ are 4 and 2, respectively. The delay parameter $\alpha$ is 2 and the system delay is
$D=2(\alpha+1) M-1=23$ samples. In order to avoid large dynamic range in the coefficients of the quotients, a similar approach as in Example 4.1 is employed. The delay parameter $d_{c}^{(i)}$ in (3-11) is therefore equal to 2 . The pairs $\left(N_{4}(z), N_{0}(z)\right)$ and $\left(N_{1}(z), N_{2}(z)\right)$ are selected to be factored, and the factorization is

$$
\begin{aligned}
& {\left[\begin{array}{cc}
N_{7}(z) & N_{3}(z) \\
-N_{4}(z) & N_{0}(z)
\end{array}\right]} \\
& \quad=\prod_{i=0}^{3}\left[\begin{array}{cc}
q_{i}^{(0)}(z) & 1 \\
1 & 0
\end{array}\right] \cdot\left[\begin{array}{cc}
L q_{4}^{(0)} & z^{-1} \\
z^{-1} & 0
\end{array}\right] \times\left[\begin{array}{cc}
K_{0} & -Q_{0}^{\prime}(z) \\
0 & -\beta / K_{0} \cdot z^{-1}
\end{array}\right],
\end{aligned}
$$

where $l=0$;

$$
\begin{aligned}
& {\left[\begin{array}{cc}
N_{2}(z) & N_{6}(z) \\
-N_{1}(z) & N_{5}(z)
\end{array}\right]} \\
& \quad=\prod_{i=0}^{3}\left[\begin{array}{cc}
q_{i}^{(1)}(z) & 1 \\
1 & 0
\end{array}\right] \cdot\left[\begin{array}{cc}
L q_{4}^{(1)} & z^{-1} \\
z^{-1} & 0
\end{array}\right] \times\left[\begin{array}{cc}
K_{1} & -Q_{1}^{\prime}(z) \\
0 & -\beta / K_{1} \cdot z^{-1}
\end{array}\right],
\end{aligned}
$$

Their coefficients are shown in Table 3. The frequency response of the analysis filter banks after factorization is shown in Figure 1(c). Implementation of (4-3) requires 5 multiplications and 4.5 additions per sample, while direct implementation of analysis polyphase matrix requires 12 multiplications and 11 additions per sample. Due to page limitation, their SOPOT counterparts are not shown here.

\section{CONCLUSION}

A new factorization for the $M$-channel PR IIR CMFB proposed in [3] is presented. It is based on the lifting scheme, and is complete for the PR FIR CMFB as well as the general twochannel PR IIR filter banks if the determinant of the polyphase matrix is equal to constant multiplies of signal delays. It can be used to convert a numerically optimized nearly PR CMFB to a structurally PR system and reduces its arithmetic complexity asymptotically by a factor of two. By using SOPOT coefficients, the multiplications in the CMFB can be replaced by simple shifts and additions, making it very attractive for VLSI implementation.

\section{REFERENCES}

[1] R. D. Koilpillai and P. P. Vaidyanathan, "Cosine-modulated FIR filter banks satisfying perfect reconstruction," IEEE Trans. on SP, pp. 770-783, Apr. 1992.

[2] H. S. Malvar, "Extended Lapped Transforms: Properties, Applications, and Fast Algorithms," IEEE Trans. SP., Vol. 40, pp. 27032714, Nov. 1992.

[3] J. S. Mao, S. C. Chan and K. L. Ho, "Theory and design of a class of $M$-channel IIR cosine-modulated filter banks," IEEE Signal Processing Letters, Feb. 2000.

[4] I. Daubechies and W. Sweldens, "Factoring wavelet transform into lifting steps," J. Fourier Anal. Appl., Vol. 4, No. 3, pp. 247-269, 1998.

[5] G. Schuller, "A new factorization and structure for cosine modulated filter banks with variable system delay," Thirtieth Asilomar Conference on Signals, Systems and Computers, vol. 2, pp. 1310-1314, 1997.

[6] X. Gao, Z. He, and X. Xin, "Efficient implementation of arbitrarylength cosine-modulated filter bank," IEEE Trans. on Signal Processing, Vol, 47, No. 4, pp. 1188-1192, April 1999.

[7] P. N. Heller, T. Karp, and T. Q. Nguyen, "A general formulation of modulated filter banks," IEEE Trans. Sig. Proc. Vol. 47, No. 4, 1999, pp. 986-1002.

[8] Y. Luo, S. C. Chan, and K. L. Ho, "Theory and Design of ArbitraryLength Biorthogonal Cosine-Modulated Filter Banks," Proc. ISCAS-97, 1997, pp. 2429-2432.

[9] T. Karp and A. Mertins, "Biorthogonal Cosine-Modulated Filters Banks without DC Leakage," Proc. IEEE ICASSP, Vol. 3, pp. 1457-1460, 1998.

[10] S. C. Chan, W. Liu, and K. L. Ho, "Perfect Reconstruction Modulated Filter Banks with Sum of Powers-of-Two Coefficients, " in Proc. IEEE ISCAS'2000, Geneva. 


\begin{tabular}{|c|c|c|}
\hline & $z^{0}$ & $z^{-1}$ \\
\hline$q_{0}^{(0)}$ & $\begin{array}{c}-4.642508093579995 \mathrm{e}- \\
001\end{array}$ & 0 \\
\hline$q_{1}^{(0)}$ & $\begin{array}{c}-2.609485580814473 \mathrm{e}- \\
002\end{array}$ & $\begin{array}{c}-1.422119789394872 \mathrm{e}- \\
001\end{array}$ \\
\hline$q_{2}^{(0)}$ & $1.041254136252027 \mathrm{e}+000$ & $\begin{array}{l}-1.634106535095274 \mathrm{e}- \\
001\end{array}$ \\
\hline$q_{3}^{(0)}$ & $\begin{array}{c}-1.752486844870494 \mathrm{e}- \\
001\end{array}$ & $\begin{array}{c}-2.824056055807716 \mathrm{e}- \\
001\end{array}$ \\
\hline$L q_{4}^{(0)}$ & $3.126535797950194 \mathrm{e}-001$ & $4.269246609153910 \mathrm{e}+000$ \\
\hline$q_{0}^{(1)}$ & $\begin{array}{c}-1.511505986777205 e- \\
001\end{array}$ & 0 \\
\hline$q_{1}^{(1)}$ & $\begin{array}{c}-5.256502606016279 \mathrm{e}- \\
001\end{array}$ & $1.550783085697046 \mathrm{e}-001$ \\
\hline$q_{2}^{(1)}$ & $\begin{array}{c}- \\
1.107633198159869 \mathrm{e}+000\end{array}$ & $2.025591660952922 \mathrm{e}-001$ \\
\hline$\overline{q_{3}^{(1)}}$ & $4.564456857968386 \mathrm{e}-001$ & $3.237301212945506 \mathrm{e}+000$ \\
\hline \multirow[t]{2}{*}{$L q_{4}$} & $4.214446420237506 \mathrm{e}-001$ & $2.170551252533108 \mathrm{e}+000$ \\
\hline & $-Q_{0}^{\prime}(z)$ & $-Q_{1}^{\prime}(z)$ \\
\hline$z^{0}$ & $\begin{array}{l}1.339338205025099 \mathrm{e}- \\
003\end{array}$ & $\begin{array}{c}-6.075680844340[14 \mathrm{e}- \\
002 \\
\end{array}$ \\
\hline$z^{-1}$ & $\begin{array}{c}1.711149850503123 \mathrm{e}- \\
001\end{array}$ & $1.117747288947271 \mathrm{e}+000$ \\
\hline \multicolumn{3}{|c|}{ Scaling factors } \\
\hline & \multirow[t]{2}{*}{$\beta$} & $26343724072108 \mathrm{e}-002$ \\
\hline & & $279543436538917 \mathrm{e}-002$ \\
\hline & $\bar{K}$ & $88614619712162 \mathrm{e}-002$ \\
\hline
\end{tabular}

Table 1. Coefficients of 4-channel FIR CMFB in example 4.1.

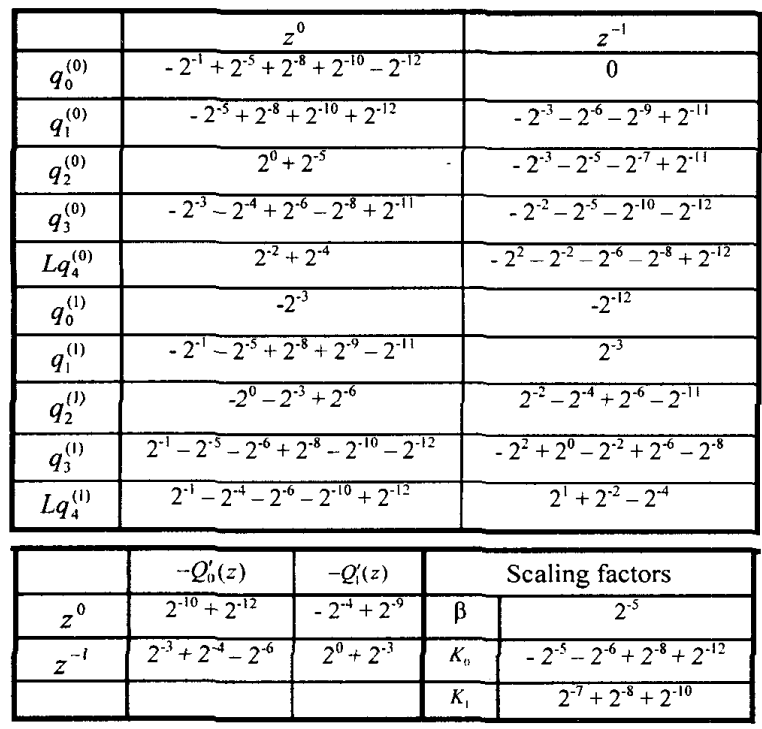

Table 2. Coefficients of multiplier-less 4-channel FIR CMFB in example 4.2.

\begin{tabular}{|c|c|c|}
\hline & $z^{0}$ & $z^{-1}$ \\
\hline$q_{0}^{(0)}$ & $3.839953359572444 \mathrm{e}-001$ & 0 \\
\hline$q_{1}^{(0)}$ & $-5.330797659340726 \mathrm{e}-001$ & $-2.561369205759393 \mathrm{e}-001$ \\
\hline$q_{2}^{(0)}$ & $1.561310374208863 \mathrm{e}+001$ & $3.493287873604430 \mathrm{e}+000$ \\
\hline$q_{3}^{(0)}$ & $-8.524163355881699 \mathrm{e}-002$ & $2.185154433785951 \mathrm{e}-001$ \\
\hline$L q_{4}^{(0)}$ & $7.622616626200014 \mathrm{e}+000$ & $2.708829156811539 \mathrm{e}+000$ \\
\hline$q_{0}^{(1)}$ & $2.273489431714420 \mathrm{e}+000$ & 0 \\
\hline
\end{tabular}

\begin{tabular}{|c|c|c|}
\hline$q_{1}^{(1)}$ & $-5.183990399531847 \mathrm{e}-001$ & $4.752664554602261 \mathrm{e}-002$ \\
\hline$q_{2}^{(1)}$ & $3.784429309928137 \mathrm{e}+000$ & $1.255986866040156 \mathrm{e}+000$ \\
\hline$q_{3}^{(1)}$ & $1.479031763369725 \mathrm{e}-001$ & $2.808848555766270 \mathrm{e}-001$ \\
\hline$L q_{4}^{(1)}$ & $-3.712245765148790 \mathrm{e}-001$ & $1.301803635739650 \mathrm{e}+000$ \\
\hline
\end{tabular}

\begin{tabular}{|c|c|c|}
\hline & $-Q_{0}^{\prime}(z)$ & $-Q_{1}^{\prime}(z)$ \\
\hline$z^{0}$ & $-2.274946795285443 \mathrm{e}-002$ & $1.100113103764122 \mathrm{e}-001$ \\
\hline$z^{-1}$ & $5.466681554977564 \mathrm{e}-001$ & $1.428649666621459 \mathrm{e}-001$ \\
\hline$z^{-2}$ & $3.579978508727065 \mathrm{e}-001$ & $6.258106529099236 \mathrm{e}-002$ \\
\hline$z^{-3}$ & $7.645476611243766 \mathrm{e}-002$ & $1.097330240803166 \mathrm{e}-002$ \\
\hline$z^{-4}$ & $5.394077803129912 \mathrm{e}-003$ & $6.762124323740777 \mathrm{e}-004$ \\
\hline
\end{tabular}

\begin{tabular}{|c|c|}
\hline \multicolumn{2}{|c|}{ Scaling factors } \\
\hline$\beta$ & $3.130693031163209 \mathrm{e}-002$ \\
\hline$K_{0}$ & $4.498469741026905 \mathrm{e}-003$ \\
\hline$K_{1}$ & $7.466808169803180 \mathrm{e}-002$ \\
\hline
\end{tabular}

Table 3. Coefficients of 4-channel IIR CMFB in example 4.3.

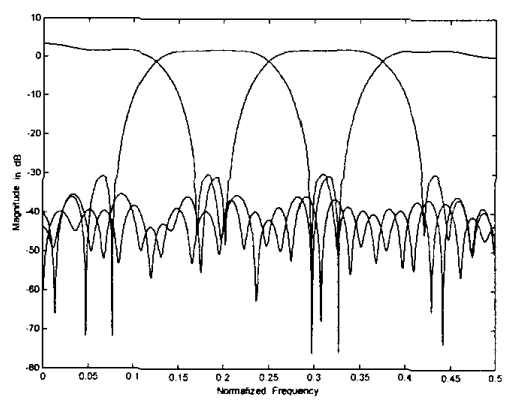

(a)

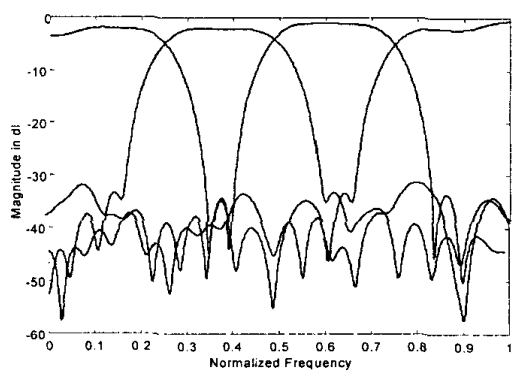

(b)

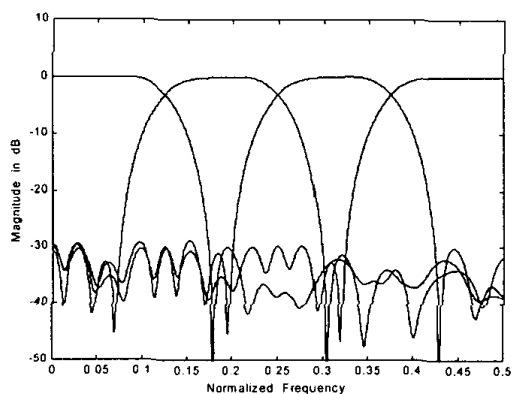

(c)

Figure 1. Frequency response of analysis filters in : (a) example 4.1, (b) example 4.2, and (c) example 4.3. 